\title{
Anisotropic inflation in the Finsler spacetime
}

\author{
Xin $\mathbf{L i}^{1,2, a}$, Sai Wang ${ }^{2, b}$, Zhe Chang ${ }^{2,3, c}$ \\ ${ }^{1}$ Department of Physics, Chongqing University, Chongqing 401331, China \\ ${ }^{2}$ State Key Laboratory of Theoretical Physics, Institute of Theoretical Physics, Chinese Academy of Sciences, Beijing 100049, China \\ ${ }^{3}$ Institute of High Energy Physics, Chinese Academy of Sciences, Beijing 100049, China
}

Received: 2 April 2015 / Accepted: 19 May 2015 / Published online: 11 June 2015

(C) The Author(s) 2015. This article is published with open access at Springerlink.com

\begin{abstract}
We suggest the universe is Finslerian in the stage of inflation. The Finslerian background spacetime breaks rotational symmetry and induces parity violation. The primordial power spectrum is given for the quantum fluctuation of the inflation field. It depends not only on the magnitude of the wavenumber but also on the preferred direction. We derive the gravitational field equations in the perturbed Finslerian background spacetime, and we obtain a conserved quantity outside the Hubble horizon. The angular correlation coefficients are presented in our anisotropic inflation model. The parity violation feature of Finslerian background spacetime requires that the anisotropic effect only appears in the angular correlation coefficients if $l^{\prime}=l+1$. The numerical results of the angular correlation coefficients are given describing the anisotropic effect.
\end{abstract}

\section{Introduction}

Cosmic inflation [1-5], as one of basic ideas of modern cosmology, plays an essential role for the very early universe. During the inflation era, the universe undergoes an exponential expansion in a very short time. Such a property of inflation successfully accounts for the flatness problem and the horizon problem [6]. Moreover, the vacuum fluctuation of the inflation field that generates the primordial energy density perturbation is a seed of cosmic anisotropy. Associated with the primordial energy density perturbation, the initial scalar perturbation is predicted to be adiabatic, Gaussian, and nearly scale invariant. The recent astronomical observations on the anisotropy of cosmic microwave background (CMB) $[7,8]$ show that the exact scale invariance of the scalar perturbation is broken with more than 5 standard deviations. The observations give stringent limits on the deviation from

\footnotetext{
a e-mail: lixin1981@cqu.edu.cn

b e-mail: wangsai@itp.ac.cn

c e-mail: changz@ihep.ac.cn
}

Gaussian statistics $[9,10]$. These facts support the idea that the inflation model is a preferred model that generates the primordial quantum fluctuations. In the standard single-field inflation model, cosmic inflation can be described by nearly de Sitter (dS) spacetime. Only six isometries are preserved in nearly dS spacetime, namely, three spatial translations and three spatial rotations. The other four isometries of dS spacetime are broken. Especially, the violation of time translation shows that the primordial power spectrum is not scale invariant. The observations on the CMB anisotropy $[7,8]$ limit the magnitude of the deviation from scale invariance to $\mathscr{O}\left(10^{-2}\right)$.

Recently, the CMB power asymmetry has been reported [11-18]. It encourages physicists to study the anisotropic inflation model, which breaks the rotational symmetry of the nearly dS spacetime. In the usual anisotropic inflation model [19-29], a primordial vector field aligned in a preferred direction is involved and the rotational symmetry is broken. In such an anisotropic inflation model, the comoving curvature perturbation becomes statistically anisotropic [30-44]. Usually, the background spacetime of anisotropic inflation model is described by the Bianchi spacetime [45]. The Planck 2013 CMB temperature map gives a limit on the deviation of Bianchi spacetime from Friedmann-RobertsonWalker (FRW) spacetime. The upper bound of the rotation invariance violation during inflation is limited to less than $\mathscr{O}\left(10^{-9}\right)$ [46-49].

In this paper, we will study anisotropic inflation in which the background spacetime is taken to be Finslerian. Finsler geometry [50] is a new geometry, which involves the Riemann geometry as its special case. Chern pointed out that Finsler geometry is just Riemann geometry without quadratic restriction, in his Notices of AMS. We choose Finsler geometry to investigate anisotropic inflation for three reasons. First, the flat Finsler spacetime, as the counterpart of Minkowski spacetime, admits less Killing vectors than Riemann spacetime does [51]. The numbers of independent Killing vectors of an $n$ dimensional non-Riemannian Finsler spacetime 
should be no more than $n(n-1) / 2+1$ [52]. It is expected that the rotational symmetry is broken in Finsler spacetime. Second, the gravitational field equation in the modified Finslerian FRW spacetime has been set up [53]. Such a Finsler spacetime can be used to investigate the cosmological preferred direction that is implied by the Union2 SnIa data $[54,55]$. Last but not the least, generally, Finsler spacetime is non-reversible under a parity flip, $\mathbf{x} \rightarrow-\mathbf{x}$. A typical nonreversible Finsler spacetime is Randers spacetime [56]. Such a property makes a function in Fourier space $\phi(-\mathbf{k})$ different from $\phi^{*}(\mathbf{k})$. Thus, one can expect that the primordial power spectrum in the Finsler spacetime violates the parity symmetry, namely, the power spectrum is not invariant under the parity flip, $\mathbf{k} \rightarrow-\mathbf{k}$.

The rest of the paper is arranged as follows: In Sect. 2, we present the anisotropic inflation model in Finsler spacetime. The background Finsler spacetime breaks the rotational symmetry and induces parity violation. We derive the primordial power spectrum for the quantum fluctuation of inflation field. In Sect. 3, we derive the gravitational field equations in the perturbed Finslerian background spacetime, and we obtain a conserved quantity outside the Hubble horizon. In Sect. 4, we derive the angular correlation coefficients in our anisotropic inflation model. We plot the numerical results of the angular correlation coefficients that describe the anisotropic effect. Conclusions and remarks are given in Sect. 5.

\section{Anisotropic inflation in the Finsler spacetime}

Finsler geometry is based on the so-called Finsler structure $F$ defined on the tangent bundle of a manifold $M$, with the property $F(x, \lambda y)=\lambda F(x, y)$ for all $\lambda>0$, where $x \in M$ represents position and $y$ represents velocity. The Finslerian metric is given as [50]

$g_{\mu \nu} \equiv \frac{\partial}{\partial y^{\mu}} \frac{\partial}{\partial y^{\nu}}\left(\frac{1}{2} F^{2}\right)$.

Throughout this paper, the indices are lowered and raised by $g_{\mu \nu}$ and its inverse matrix $g^{\mu \nu}$.

The Finslerian metric reduces to the Riemannian metric, if $F^{2}$ is quadratic in $y$.

In order to describe the anisotropic inflation, we propose that the background Finsler spacetime is of the form

$F_{0}^{2}=y^{t} y^{t}-a^{2}(t) F_{\mathrm{Ra}}^{2}$,

where $F_{\mathrm{Ra}}$ is a Randers space [56],

$F_{\mathrm{Ra}}=\alpha+\beta=\sqrt{\delta_{i j} y^{i} y^{j}}+\delta_{i j} b^{i} y^{j}$,

$\alpha$ denotes the Riemannian metric, and $\beta$ denotes the one form. Here, we require that the vector $b^{i}$ in $F_{\mathrm{Ra}}$ is of the form $b^{i}=\{0,0, b\}$ and $b$ is a constant. It is obvious that the background spacetime (2) returns to FRW spacetime when $b=0$. The spatial part of the universe $F_{\mathrm{Ra}}$ is a flat Finsler space, since all types of Finslerian curvature vanish for $F_{\mathrm{Ra}}$. The Killing equations of the Randers space $F_{\mathrm{Ra}}$ are given as [51,57]

$L_{V} \alpha=\delta_{k j} \frac{\partial V^{k}}{\partial x^{i}}+\delta_{k i} \frac{\partial V^{k}}{\partial x^{j}}=0$,

$L_{V} \beta=b \frac{\partial V^{z}}{\partial x^{i}}=0$,

where $L_{V}$ is the Lie derivative along the Killing vector $V$. Noticing that the vector $b^{i}$ is parallel to the $z$-axis, we find from the Killing equations (4) and (4) that there are four independent Killing vectors in the Randers space $F_{\mathrm{Ra}}$. Three of them represent the translation symmetry, and the rest one represents the rotational symmetry in the $x-y$ plane. It means that the rotational symmetries in the $x-z$ and the $y-z$ planes are broken. Also, the spatial part of Finsler spacetime (2) is non-reversible for $z \rightarrow-z$. It means that the party is violated in the Finsler spacetime (2).

In our anisotropic inflation model, we just consider the single-field "slow-roll" inflation. The action for this scalar field is given by

$S=\int \mathrm{d}^{4} x \sqrt{-g}\left(\frac{1}{2} g^{\mu \nu} \partial_{\mu} \phi \partial_{\nu} \phi-V(\phi)\right)$.

The quantum fluctuation $[58,59]$ is associated with the firstorder perturbation $\delta \phi(t, \mathbf{x})$ of the inflation field $\phi$. Noticing that the determinant of the background spacetime (2) is given by $g=-a^{3} F_{\mathrm{Ra}}^{3} /\left(\delta_{i j} y^{i} y^{j}\right)^{3 / 2}$, we find from the action (6) that the equation of motion for $\delta \phi$ is given as

$\delta \ddot{\phi}+3 H \delta \dot{\phi}-a^{-2} \bar{g}^{i j} \partial_{i} \partial_{j} \delta \phi=0$,

where the dot denotes the derivative with respect to time, $H \equiv \dot{a} / a$, and $\bar{g}^{i j}$ is the Finslerian metric for the Rander space $F_{\mathrm{Ra}}$. The Finslerian metric $\bar{g}^{i j}$ is a homogeneous function of degree 0 with respect to the variable $y$. It means that the Finslerian metric $\bar{g}^{i j}$ depends on direction. Such a property makes the solution of the equation of motion (7) much more complicated. In this paper, for simplicity, we take the direction of $y$ to be parallel with the wave vector $\mathbf{k}$ in the momentum space. Therefore, in the momentum space, Eq. (7) can be simplified as

$\delta \ddot{\phi}+3 H \delta \dot{\phi}-a^{-2} k_{e}^{2} \delta \phi=0$,

where the effective wavenumber $k_{e}$ is given by

$k_{e}^{2}=\bar{g}^{i j} k_{i} k_{j}=k^{2}\left(1+b \hat{\mathbf{k}} \cdot \hat{\mathbf{n}}_{z}\right)^{2}$. 
Then, following the standard quantization process in the inflation model [60], we can obtain the primordial power spectrum from the solution of Eq. (8). It is of the form

$\mathscr{P}_{\delta \phi}(\mathbf{k})=\mathscr{P}_{0}(k)\left(\frac{k}{k_{e}}\right)^{3} \simeq \mathscr{P}_{0}\left(1-3 b \hat{\mathbf{k}} \cdot \hat{\mathbf{n}}_{z}\right)$,

where $\mathscr{P}_{0}$ is an isotropic power spectrum for $\delta \phi$ which depends only on the magnitude of wavenumber $k$. The term $3 b \hat{\mathbf{k}} \cdot \hat{\mathbf{n}}_{z}$ in the primordial power spectrum $\mathscr{P}_{\delta \phi}$ represents the effect of rotational symmetry breaking.

\section{Gravitational field equation in anisotropic inflation}

At the end stage of inflation, the background metric perturbation is coupled with the inflation field. Thus the initial power spectrum differs from $\mathscr{P}_{\delta \phi}$. In the standard inflation model, the comoving curvature perturbation that links the inflation field with the background metric perturbation is conserved outside the Hubble horizon. In our anisotropic inflation model, we should find a counterpart of the comoving curvature perturbation.

We start from investigating the gravitational field equation for the perturbation of the Finsler structure (2). In this paper, we just consider the scalar perturbation. For simplicity, the scalar perturbed Finsler structure is taken to be of the form

$F^{2}=(1+2 \Psi(t, \mathbf{x})) y^{t} y^{t}-a^{2}(t)(1+2 \Phi(t, \mathbf{x})) F_{\mathrm{Ra}}^{2}$,

where $\Psi$ and $\Phi$ are scalar perturbations. In Finsler geometry, there is a geometrical invariant quantity, i.e., the Ricci scalar. It is of the form [50]

$$
\begin{aligned}
\text { Ric } \equiv & \frac{1}{F^{2}}\left(2 \frac{\partial G^{\mu}}{\partial x^{\mu}}-y^{\lambda} \frac{\partial^{2} G^{\mu}}{\partial x^{\lambda} \partial y^{\mu}}+2 G^{\lambda} \frac{\partial^{2} G^{\mu}}{\partial y^{\lambda} \partial y^{\mu}}\right. \\
& \left.-\frac{\partial G^{\mu}}{\partial y^{\lambda}} \frac{\partial G^{\lambda}}{\partial y^{\mu}}\right),
\end{aligned}
$$

where $G^{\mu}$ is for the geodesic spray coefficients,

$G^{\mu}=\frac{1}{4} g^{\mu \nu}\left(\frac{\partial^{2} F^{2}}{\partial x^{\lambda} \partial y^{\nu}} y^{\lambda}-\frac{\partial F^{2}}{\partial x^{\nu}}\right)$.

The Ricci scalar only depends on the Finsler structure $F$ and is insensitive to the connections. Plugging the perturbed Finsler structure (11) into the formula of the Ricci scalar (12), we obtain

$$
\begin{aligned}
& F^{2} \operatorname{Ric}=-3 \frac{\ddot{a}}{a} y^{t} y^{t}+\left(a \ddot{a}+2 \dot{a}^{2}\right) F_{\mathrm{Ra}}^{2} \\
& +y^{t} y^{t}\left(a^{-2} \bar{g}^{i j} \Psi_{, i, j}-3 \ddot{\Phi}+3 H(\dot{\Psi}-2 \dot{\Phi})\right) \\
& +y^{t} y^{i}\left(4 H \Psi_{, i}-4 \dot{\Phi}_{, i}\right)-y^{i} y^{j}\left(\Psi_{, i, j}+\Phi_{, i, j}\right) \\
& +F_{\mathrm{Ra}}^{2}\left(\left(2 \dot{a}^{2}+a \ddot{a}\right)(2 \Phi-2 \Psi)+a \dot{a}(6 \dot{\Phi}-\dot{\Psi})\right. \\
& \left.+a^{2} \ddot{\Phi}-\bar{g}^{i j} \Phi_{, i, j}\right) \\
& +\frac{1}{2} \frac{\partial \bar{g}^{i j}}{\partial y^{i}}\left(\Phi_{, j, k} y^{k}+\dot{\Phi}_{, j} y^{t}+2 H \Psi_{, j} y^{t}\right) F_{\mathrm{Ra}}^{2} \\
& -\frac{1}{2 a^{2}} \frac{\partial \bar{g}^{i j}}{\partial y^{i}}\left(\Psi_{, j, k} y^{k}+\dot{\Psi}_{, j} y^{t}+2 H \Psi_{, j} y^{t}\right) y^{t} y^{t},
\end{aligned}
$$

where the comma denotes the derivative with respect to the spatial coordinate $x$, and $H \equiv \dot{a} / a$. In Sect. 2, we have taken the direction of $y^{i}$ to be parallel with the wave vector $\mathbf{k}$ in momentum space. Noticing the relation $\frac{\partial \bar{g}^{i j}}{\partial y^{i}} y_{j}=0$, we find that the term of Eq. (14) which is proportional to $\frac{\partial \bar{g}^{i j}}{\partial y^{i}}$ should vanish in momentum space. In Refs. [53,57], we have proved that the gravitational field equation in the Finsler spacetime is of the form

$G_{v}^{\mu}=8 \pi G T_{v}^{\mu}$,

where the modified Einstein tensor in the Finsler spacetime is defined as

$G_{v}^{\mu} \equiv \operatorname{Ric}_{v}^{\mu}-\frac{1}{2} \delta_{v}^{\mu} S$

and $T_{v}^{\mu}$ is the energy-momentum tensor. Here the Ricci tensor is defined as [61]

$\operatorname{Ric}_{\mu \nu}=\frac{\partial^{2}\left(\frac{1}{2} F^{2} \operatorname{Ric}\right)}{\partial y^{\mu} \partial y^{\nu}}$,

and the scalar curvature in the Finsler spacetime is given as $S=g^{\mu \nu} R i c_{\mu \nu}$. Plugging the equation for the Ricci scalar (14) into the gravitational field equation, we obtain the background equations

$3 H^{2}=8 \pi G T_{(0) 0}^{0}$,

$\frac{2 \ddot{a}}{a}+H^{2}=-8 \pi G T_{(0) i}^{i} / 3$,

and the perturbed equations in momentum space,

$$
\begin{aligned}
& 6 H \dot{\Phi}-6 H^{2} \Psi+2 \frac{k_{e}^{2} \Phi}{a^{2}}=8 \pi G \delta T_{0}^{0} \\
& -i(2 H \Psi-2 \dot{\Phi}) k_{i}=8 \pi G \delta T_{i}^{0}, \\
& 2 \ddot{\Phi}+H(6 \dot{\Phi}-2 \dot{\Psi})-\Psi\left(2 H^{2}+4 \frac{\ddot{a}}{a}\right)+\frac{k_{e}^{2}}{a^{2}}(\Phi+\Psi) \\
& =8 \pi G \delta T_{i}^{i} / 3
\end{aligned}
$$


$-a^{-2} k^{i} k_{j}(\Phi+\Psi)=8 \pi G \delta T_{j}^{i} \quad(i \neq j)$,

where $k^{i}=\bar{g}^{i j} k_{j}$. Here, the energy-momentum tensor in the above field equations is derived by the variation of the action (6) of inflation field $\phi$. It is of the form

$T_{\mu \nu}=\partial_{\mu} \phi \partial_{\nu} \phi-g_{\mu \nu}\left(\frac{1}{2} g^{\alpha \beta} \partial_{\alpha} \phi \partial_{\beta} \phi-V(\phi)\right)$,

where $T_{(0) 0}^{0}$ and $T_{(0) i}^{i}$ in Eqs. (18) and (19) correspond to zeroth-order part of inflation field, i.e. $\phi_{0}(t)$, and $\delta T_{\nu}^{\mu}$ in Eqs. (20)-(23) corresponds to first-order part of inflation field, i.e. $\delta \phi(t, \mathbf{x})$. Now, we find that the background equations (18) and (19) are the same as the standard inflation model that means our universe is exponentially expanding if the slow-roll condition $\dot{\phi}_{0} \ll V\left(\phi_{0}\right)$ is satisfied. However, one should notice that there is difference between standard inflation model and our anisotropic inflation model. During the expansion, the spatial form of universe is Euclidean in standard inflation model. In our anisotropic inflation model, the spatial form of universe is Finslerian. This anisotropic feature is obvious in the perturbed equations. The perturbed equations (20)-(23) are the same as the standard inflation model except for replacing wavenumber $k$ with effective wavenumber $k_{e} . k_{e}$ depends not only on the magnitude of $k$ but also the preferred direction $\hat{\mathbf{n}}_{z}$, which induces rotational symmetry breaking.

Following the approach of standard inflation model [60], we find from the field equations (18)-(23) that

$k_{e}^{2} \Phi \propto\left(\mathscr{H} \frac{\delta \phi}{\phi_{0}^{\prime}}-\Phi\right)^{\prime}$,

where the prime denotes the derivative with respect to conformal time $\eta \equiv \int \frac{\mathrm{d} t}{a}$ and $\mathscr{H} \equiv \frac{a^{\prime}}{a}$. During the stage of inflation, we are interested in the modes of quantum fluctuations that are outside the Hubble horizon. Therefore, the terms that are proportional to $k^{2}$ can be ignored. Then,we find from Eq. (25) that the comoving curvature perturbation $R_{c} \equiv \mathscr{H} \frac{\delta \phi}{\phi_{0}^{\prime}}-\Phi$ is conserved outside the Hubble horizon. The comoving curvature perturbation $R_{C}$ is the same as that in the standard inflation model. This is due to the fact that $R_{c}$ does not depend on the wavenumbers $k$, such that the anisotropic effect does not appear explicitly in $R_{c}$. Thus, we can use Eq. (10) to get the primordial power spectrum for $R_{c}$. It is of the form

$\mathscr{P}_{R_{c}}(\mathbf{k})=\mathscr{P}_{\text {iso }}(k)\left(1-3 b \hat{\mathbf{k}} \cdot \hat{\mathbf{n}}_{z}\right)$,

where $\mathscr{P}_{\text {iso }}$ is the isotropic power spectrum for $R_{c}$.

Technically, the term obtained in the power spectrum (26) is a dipole in Fourier space. However, it is shown in Ref. [62] that, due to the reality condition, the power spectrum $P(k)$ in
Fourier space cannot have odd $\ell$ in an harmonic expansion. It should be noticed that the definition of the Fourier transform depends on the space metric in high dimensions. In Riemann space, the Fourier transform is defined as

$f(\mathbf{k})=\int \mathrm{d}^{3} x f(\mathbf{x}) \exp \left(-i \delta_{i j} k^{i} x^{j}\right)$,

where $\delta_{i j}$ is the Euclidean metric. This expression is well defined in Riemann space, since at any fixed point one can erect a local coordinate system such that the metric is Euclidean. For any real field $f(\mathbf{x})$, one can find from the definition (27) that $f(-\mathbf{k})=f^{*}(\mathbf{k})$. Thus, Ref. [62] concludes that the power spectrum should satisfy $P(\mathbf{k})=P(-\mathbf{k})$.

However, such a conclusion is not valid in the Finsler space. In Finsler space, the metric does not have a quadratic restriction. Thus, the Finsler metric depends on the fiber coordinate $y$ in general. We have taken the direction of $y^{i}$ parallel with the wave vector $\mathbf{k}$ in the momentum space. Therefore, the Fourier transform in the Finsler space is defined as

$f(\mathbf{k})=\int \mathrm{d}^{3} x f(\mathbf{x}) \exp \left(-i \eta_{i j}(k) k^{i} x^{j}\right)$,

where $\eta_{i j}$ is the Finsler metric. The Fourier transform (28) implies that the wave equation in Finsler spacetime satisfies $\eta^{\mu \nu}(k) k_{\mu} k_{v}=0$ in vacuum. We have found that the gravitational wave in vacuum does satisfy this relation [63]. That is why we defined the Fourier transform as (28). In this paper, we consider the spatial part of Finsler spacetime to be of the form in Eq. (3). It is obvious that the metric $\eta_{i j}=\frac{\partial}{\partial y^{i}} \frac{\partial}{\partial y^{j}}\left(\frac{1}{2} F_{\mathrm{Ra}}^{2}\right)$ satisfies

$\eta_{i j}(-\mathbf{k}) \neq \eta_{i j}(\mathbf{k})$

in momentum space. For the Finsler metric does not have a quadratic restriction, one cannot erect a local coordinate system such that $F_{\mathrm{Ra}}(\mathbf{k})=F_{\mathrm{Ra}}(-\mathbf{k})$. Thus, for any real field, the relation $f(-\mathbf{k})=f^{*}(\mathbf{k})$ does not hold in Finsler space. It implies that the power spectrum $P(k)$ in Fourier space can have odd $\ell$ in harmonic expansion.

\section{Contributions to $\mathrm{CMB}$ power spectra from anisotropic inflation model}

The anisotropic term in Eq. (26) could give an off-diagonal angular correlation for the CMB temperature fluctuation and E-mode polarization. The general angular correlation coefficients that describe the anisotropic effect are given by $C_{X X^{\prime}, l l^{\prime}, m m^{\prime}}$ [64-66], where $X$ denotes $T$ and $E$, respectively. In our anisotropic model, by making use of Eq. (26), 
we obtain the CMB correlation coefficients for scalar perturbations as follows:

$$
C_{X X^{\prime}, l l^{\prime}, m m^{\prime}}=\int \frac{\ln k}{(2 \pi)^{3}} \Delta_{X, l 0}(k) \Delta_{X^{\prime}, l^{\prime} 0}^{*}(k) P_{l l^{\prime} m m^{\prime}},
$$

where

$$
\begin{aligned}
P_{l l^{\prime} m m^{\prime}} & =\int \mathrm{d} \Omega \mathscr{P}_{R_{c}} Y_{l m}^{*} Y_{l^{\prime} m^{\prime}} \\
& =\mathscr{P}_{\mathrm{iso}} \delta_{m m^{\prime}}\left(\delta_{l l^{\prime}}-3 b \sqrt{\frac{2 l^{\prime}+1}{2 l+1}} \mathscr{C}_{10 l m}^{l^{\prime} m} \mathscr{C}_{10 l 0}^{l^{\prime} 0}\right)
\end{aligned}
$$

$\Delta_{X, l s}(k)$ denote the transfer functions and $\mathscr{C}_{L M l^{\prime} m^{\prime}}^{l m}$ are the Clebsch-Gordan coefficients. Then we find from Eq. (30) that the CMB correlation coefficients $C_{l l^{\prime}}$ contain diagonal part which means $l^{\prime}=l$ and off-diagonal part which means $l^{\prime}=l+1$. Here, we have defined $C_{l l^{\prime}} \equiv C_{X X^{\prime}, l l^{\prime}, m^{\prime}}$. The diagonal part of $C_{l l^{\prime}}$, which gives the contribution of statistical isotropy, equals the $\mathrm{CMB}$ correlation coefficients in the standard cosmological model. The off-diagonal part of $C_{l l^{\prime}}$, which comes from the anisotropic term $3 b \hat{\mathbf{k}} \cdot \hat{\mathbf{n}}_{z}$ describes the deviation from statistical isotropy. One should notice that the anisotropic term $3 b \hat{\mathbf{k}} \cdot \hat{\mathbf{n}}_{z}$ in our model is not invariant under parity flip, $\mathbf{k} \rightarrow-\mathbf{k}$. Thus, the anisotropic effect in our model does not contribute to the diagonal part of $C_{l l^{\prime}}$.

The parameter $b$ in the above equation represents the amplitude of rotational symmetry violation. Currently, Planck satellite does not give direct observational bounds on CMB correlation coefficients $C_{l l+1}$. Recently, Ref. [67] used the power asymmetry of $\mathrm{CMB}$ temperature fluctuations $[11,12]$ to give a restriction on $C_{l l+1}$. The ratio of $C_{l l+1}$ to the power $C_{l}$ is found to be roughly around 0.02 for $l=2$ and decays for larger $l$ values. The amplitude of power asymmetry of CMB $[11,12]$ is of the order $10^{-2}$. Thus, our consideration for $b \sim 10^{-2}$ is valid at present. Further investigation for the dipolar model will make progress if the observational constraints for $C_{l l+1}^{T T}, C_{l l+1}^{T E, E T}$ and $C_{l l+1}^{E E}$ are given.

Here, by making use of the formula of angular correlation coefficients (30) and the Planck 2013 data [8], we plot numerical results for the off-diagonal part of $C_{l l^{\prime}}$ via modifying the Code for Anisotropies in the Microwave Background (CAMB) $[68,69]$. The off-diagonal part of $C_{l l^{\prime}}$ has two properties that differ from the diagonal part. One is that the $T E$ and $E T$ correlation coefficients are different. The other shows that the off-diagonal part of $C_{l l^{\prime}}$ depends on $m$. These properties are obvious in the following figures. The off-diagonal $T T, T E, E T, E E$ correlation coefficients for $m=0$ are shown in Figs.1, 3, 5 and 7, respectively. The off-diagonal $T T, T E, E T, E E$ correlation coefficients for $m=l$ are shown in Figs. 2, 4, 6 and 8, respectively. Here, we have used the mean value of cosmological parameters [8] to give the above figures. The coeffi-

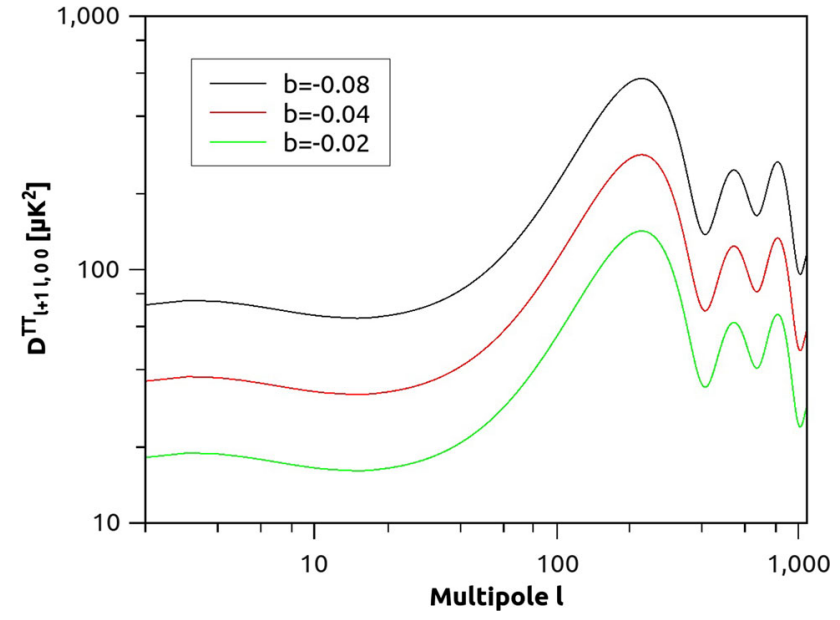

Fig. 1 The off-diagonal $T T$ correlation coefficients with $m=0$. The black, red, and green curve correspond to a different Finslerian parameter, i.e. $b=-0.08,-0.04,-0.02$, respectively. The following figures are given by the same convention

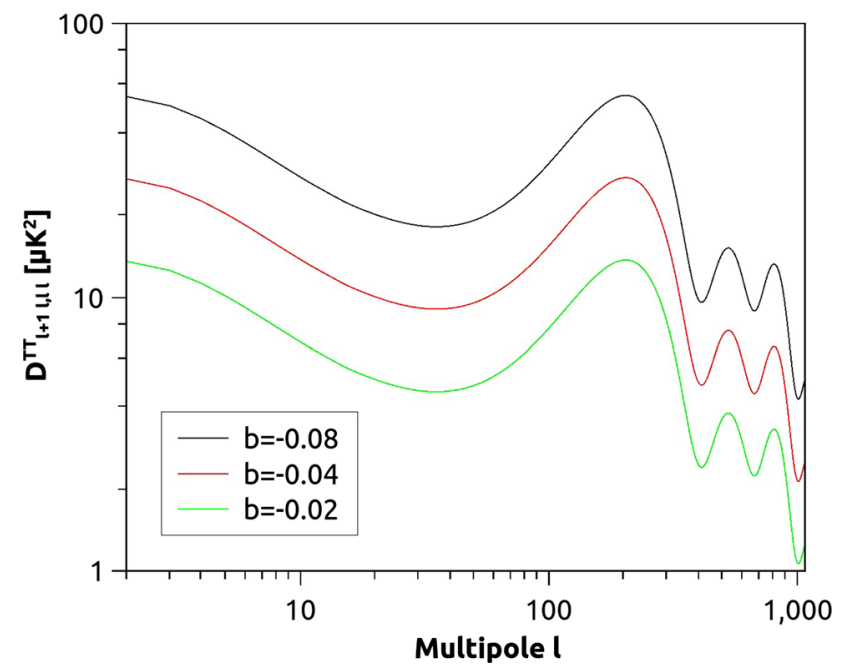

Fig. 2 The off-diagonal $T T$ correlation coefficients with $m=l$. It demonstrates that the off-diagonal correlation coefficients $C_{l l^{\prime}}$ depend on $m$

cients $D_{l l^{\prime}, m m^{\prime}}^{X X^{\prime}}$ in these figures are defined as $D_{l l^{\prime}, m m^{\prime}}^{X X^{\prime}} \equiv$ $\sqrt{l(l+1) l^{\prime}\left(l^{\prime}+1\right)} C_{X X^{\prime}, l l^{\prime}, m m^{\prime}} / 2 \pi$.

The above figures show that the off-diagonal correlation coefficients for $m=0$ have a similar shape to the diagonal part of angular correlation coefficients. This is due to the fact that the transfer function $\Delta_{l}$ approximately equals $\Delta_{l+1}$ for high $l$. In other words, they are related to nearly same physical scales.

\section{Conclusions and remarks}

In this paper, we have proposed an anisotropic inflation model in Finsler spacetime. Finsler spacetime (2) breaks the rota- 


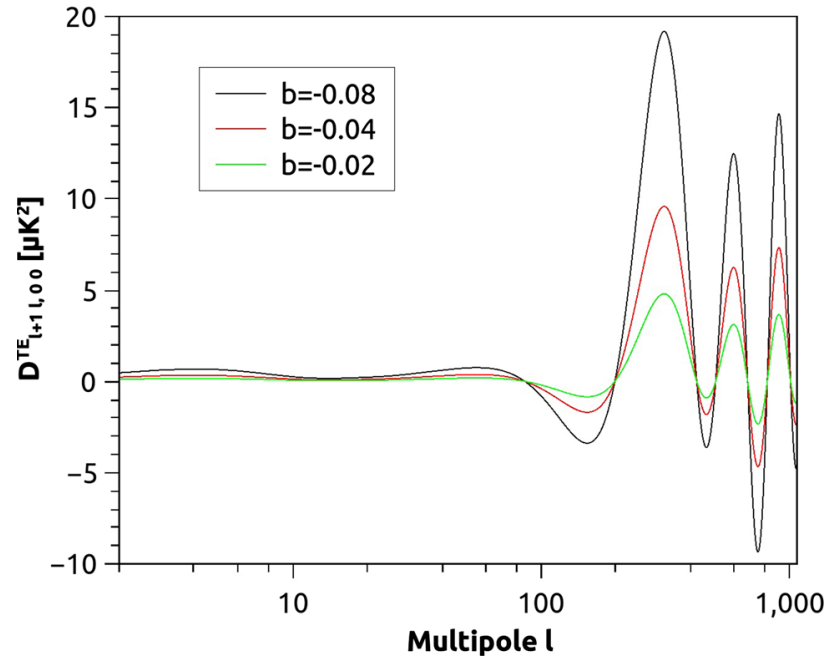

Fig. 3 The off-diagonal $T E$ correlation coefficients with $m=0$

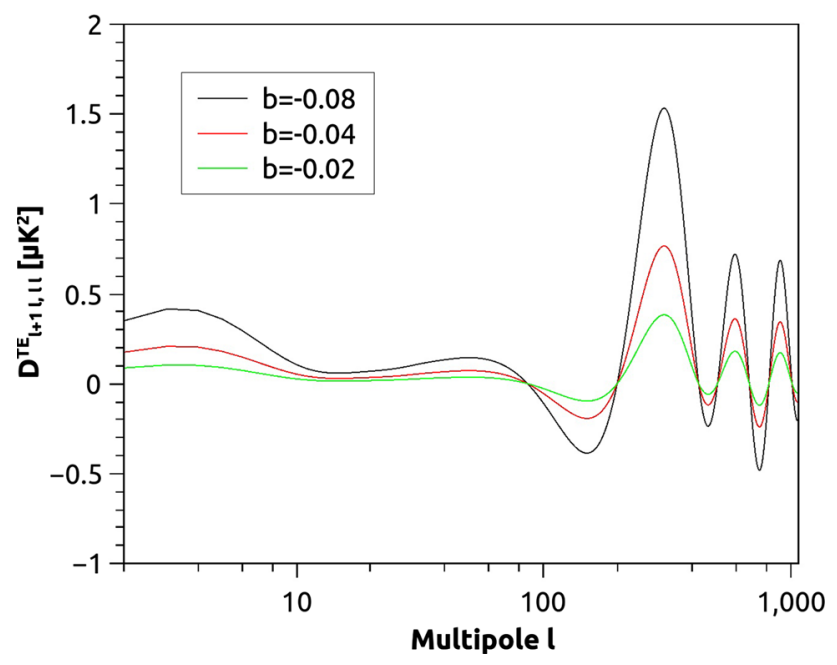

Fig. 4 The off-diagonal $T E$ correlation coefficients with $m=l$

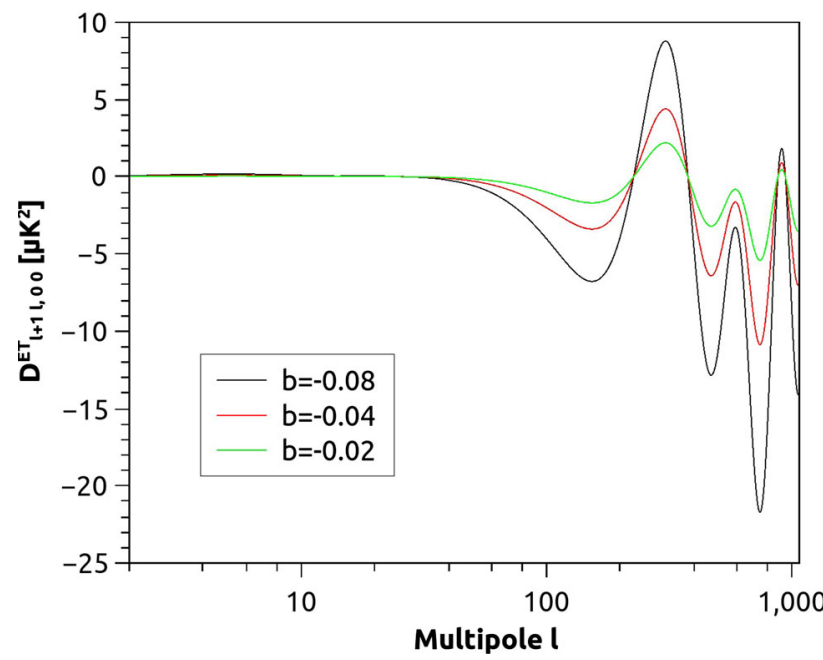

Fig. 5 The off-diagonal $E T$ correlation coefficients with $m=0$

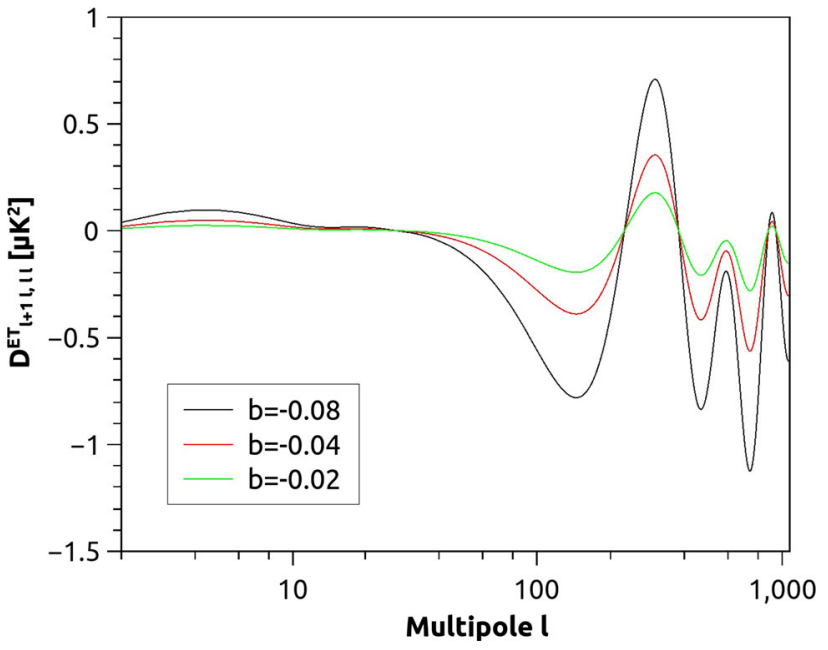

Fig. 6 The off-diagonal ET correlation coefficients with $m=l$

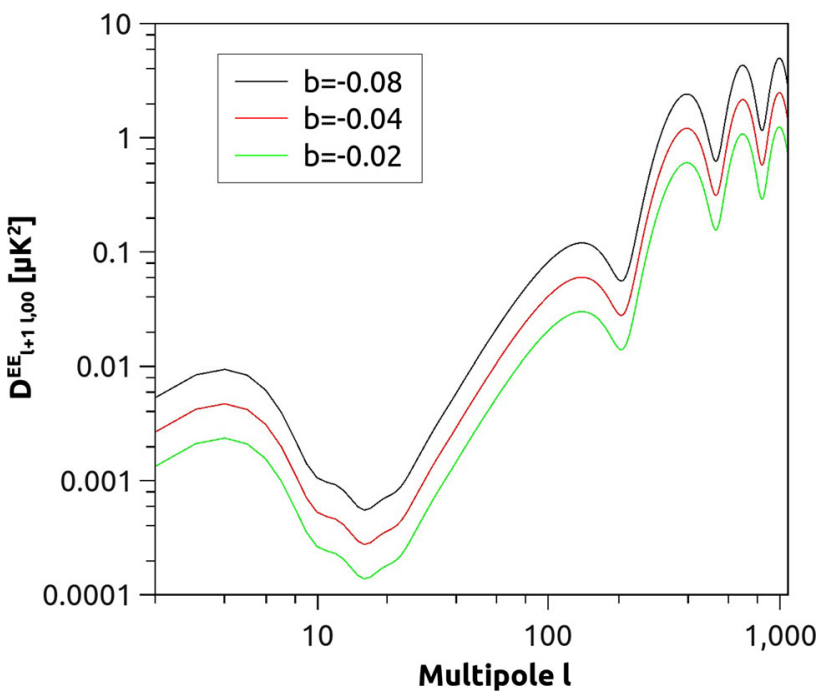

Fig. 7 The off-diagonal $E E$ correlation coefficients with $m=0$

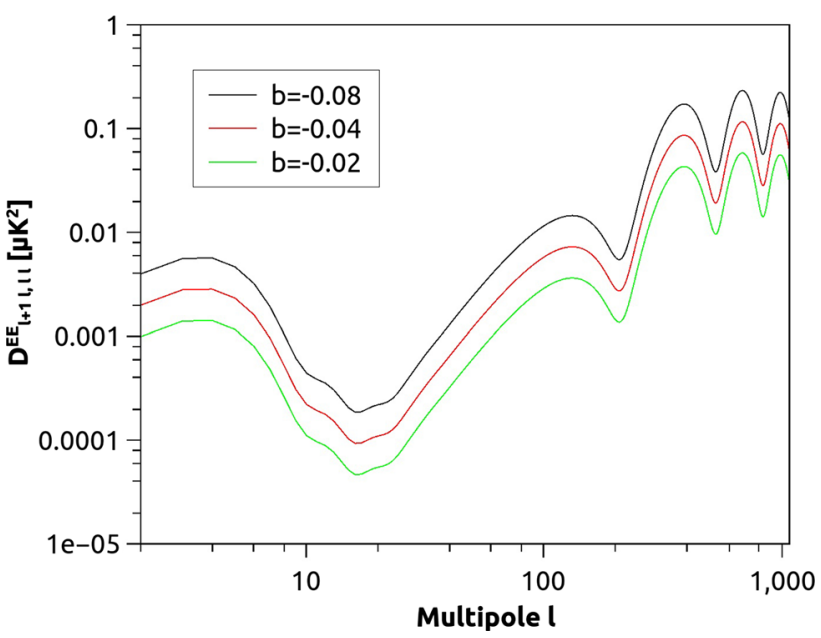

Fig. 8 The off-diagonal $E E$ correlation coefficients with $m=l$ 
tional symmetry and induces parity violation. The primordial power spectrum was obtained for the quantum fluctuation of inflation field (10). The term $3 b \hat{\mathbf{k}} \cdot \hat{\mathbf{n}}_{z}$ in the primordial power spectrum $\mathscr{P}_{\delta \phi}$ represents the effect of rotational symmetry breaking. The gravitation field equations of the perturbed Finsler spacetime (11) were presented. We have taken the direction of $y^{i}$ to be parallel with the wave vector $\mathbf{k}$ in momentum space. Thus, the dynamic feature of Finsler spacetime vanishes. The perturbed equations (20)-(23) are the same as in the standard inflation model except for replacing the wavenumber $k$ with an effective wavenumber $k_{e} . k_{e}$ depends not only on the magnitude of $k$ but also the preferred direction $\hat{\mathbf{n}}_{z}$, which represents rotational symmetry breaking. These field equations guarantee that the comoving curvature perturbation $R_{c} \equiv \mathscr{H} \frac{\delta \phi}{\phi_{0}^{\prime}}-\Phi$ is conserved outside the Hubble horizon. The comoving curvature perturbation $R_{c}$ is the same as in the standard inflation model. This is due to the fact that $R_{C}$ does not explicitly depends on the wavenumber $k$, such that the anisotropic effect does not appear in $R_{c}$. We have used the primordial power spectrum of $R_{C}$ (26) to derive the general angular correlation coefficients $C_{X X^{\prime}, l l^{\prime}, \mathrm{mm}^{\prime}}$ in our anisotropic inflation model. The parity violation feature requires that the anisotropic effect only appears in angular correlation coefficients with $l^{\prime}=l+1$, and does not contribute to the diagonal $C_{l l}$, i.e. the power spectrum. It means that the off-diagonal part of angular correlation coefficients represents the anisotropic effect. The numerical results for the off-diagonal correlation coefficients show that they depend on $m$, and the $T E$ and $E T$ correlation coefficients are different.

Acknowledgments We thank Prof. Q.-G. Huang, Dr. S.-Y. Li, and M.S. D. Zhao for useful discussions. This work has been partly funded by the National Natural Science Fund of China (NSFC) (Grant Nos. 11375203 and 11305181), and partly supported by the project of Knowledge Innovation Program of Chinese Academy of Sciences and grants from NSFC (Grant Nos. 11322545 and 11335012).

Open Access This article is distributed under the terms of the Creative Commons Attribution 4.0 International License (http://creativecomm ons.org/licenses/by/4.0/), which permits unrestricted use, distribution, and reproduction in any medium, provided you give appropriate credit to the original author(s) and the source, provide a link to the Creative Commons license, and indicate if changes were made. Funded by SCOAP ${ }^{3}$.

\section{References}

1. A.A. Starobinsky, Phys. Lett. B 91, 99 (1980)

2. K. Sato, Mon. Not. R. Astron. Soc. 195, 467 (1981)

3. A.H. Guth, Phys. Rev. D 23, 347 (1981)

4. A.D. Linde, Phys. Lett. B 108, 389 (1982)

5. A. Albrecht, P.J. Steinhardt, Phys. Rev. Lett. 48, 1220 (1982)

6. A. Riotto, arXiv:hep-ph/0210162

7. W.M.A.P. Collaboration, Astrophys. J. Suppl. 208, 19 (2013)

8. Planck Collaboration, Astron. Astrophys. 571, A16 (2014)
9. W.M.A.P. Collaboration, Astrophys. J. Suppl. 208, 20 (2013)

10. Planck Collaboration, Astron. Astrophys. 571, A24 (2014)

11. Planck Collaboration, Astron. Astrophys. 571, A23 (2014)

12. W.M.A.P. Collaboration, Astrophys. J. Suppl. 208, 20 (2013)

13. Y. Akrami, Y. Fantaye, A. Shafieloo, H.K. Eriksen, F.K. Hansen, A.J. Banday, K.M. Glőrski, Astrophys. J. 784, L42 (2014)

14. H.K. Eriksen, F.K. Hansen, A.J. Banday, K.M. Gorski, P.B. Lilje, Astrophys. J. 605, 14 (2004) [Erratum-ibid. 609, 1198 (2004)]

15. F.K. Hansen, A.J. Banday, K.M. Gorski, Mon. Not. R. Astron. Soc. 354, 641 (2004)

16. D.J. Schwarz, G.D. Starkman, D. Huterer, C.J. Copi, Phys. Rev. Lett. 93, 221301 (2004)

17. C. Gordon, W. Hu, D. Huterer, T. Crawford, Phys. Rev. D 72, $103002(2005)$

18. C.-G. Park, Mon. Not. R. Astron. Soc. 349, 313 (2004)

19. E. Dimastrogiovanni, N. Bartolo, S. Matarrese, A. Riotto, Adv. Astron. 2010, 752670 (2010)

20. A. Maleknejad, M. Sheikh-Jabbari, J. Soda, Phys. Rep. 528, 161 (2013)

21. R. Namba, Phys. Rev. D 86, 083518 (2012)

22. R. Emami, H. Firouzjahi, JCAP 1310, 041 (2013)

23. J. Soda, Class. Q. Grav. 29, 083001 (2012)

24. A.E. Gumrukcuoglu, B. Himmetoglu, M. Peloso, Phys. Rev. D 81, 063528 (2010)

25. X. Chen, Y. Wang, JCAP 1410, 027 (2014)

26. X. Chen, Y. Wang, JCAP 1407, 004 (2014)

27. L. Campanelli, P. Cea, L. Tedesco, Phys. Rev. Lett. 97, 131302 (2006)

28. L. Campanelli, P. Cea, L. Tedesco, Phys. Rev. D 76, 063007 (2007)

29. J. Kim, P. Naselsky, JCAP 07, 041 (2009)

30. L. Ackerman, S.M. Carroll, M.B. Wise, Phys. Rev. D 75, 083502 (2007)

31. M.-A. Watanabe, S. Kanno, J. Soda, Prog. Theor. Phys. 123, 1041 (2010)

32. N. Barnaby, R. Namba, M. Peloso, Phys. Rev. D 85, 123523 (2012)

33. N. Bartolo, S. Matarrese, M. Peloso, A. Ricciardone, Phys. Rev. D 87, 023504 (2013)

34. M. Shiraishi, E. Komatsu, M. Peloso, N. Barnaby, JCAP 1305, 002 (2013)

35. M. Shiraishi, E. Komatsu, M. Peloso, JCAP 1404, 027 (2014)

36. A.A. Abolhasani, R. Emami, J.T. Firouzjaee, H. Firouzjahi, JCAP 1308, 016 (2013)

37. R. Emami, H. Firouzjahi, M. Zarei, Phys. Rev. D 90, 023504 (2014)

38. P.K. Rath, P. Jain, Phys. Rev. D 91, 023515 (2015)

39. M. Zarei, arXiv:1412.0289 [hep-th]

40. Y.F. Cai, W. Zhao, Y. Zhang, Phys. Rev. D 89(2), 023005 (2014)

41. A.R. Pullen, M. Kamionkowski, Phys. Rev. D 76, 103529 (2007)

42. D.H. Lyth, M. Karciauskas, JCAP 1305, 011 (2013)

43. X. Chen, R. Emami, H. Firouzjahi, Y. Wang, JCAP 1408, 027 (2014)

44. S. Jazayeri, Y. Akrami, H. Firouzjahi, A.R. Solomon, Y. Wang, JCAP 1411, 044 (2014)

45. K. Rosquist, R.T. Jantzen, Phys. Rep. 166, 89 (1988)

46. J. Kim, E. Komatsu, Phys. Rev. D 88, 101301(R) (2013)

47. A. Naruko, E. Komatsu, M. Yamaguchi, arXiv:1411.5489 [astroph.CO]

48. S.R. Ramazanov, G. Rubtsov, Phys. Rev. D 89(4), 043517 (2014)

49. G.I. Rubtsov, S.R. Ramazanov, Phys. Rev. D 91, 043514 (2015)

50. D. Bao, S.S. Chern, Z. Shen, An Introduction to Riemann-Finsler Geometry, Graduate Texts in Mathematics 200 (Springer, New York, 2000)

51. X. Li, Z. Chang, Differ. Geom. Appl. 30, 737 (2012)

52. H.C. Wang, J. Lond. Math. Soc. s1-22(1), 5 (1947)

53. X. Li, H.-N. Lin, S. Wang, Z. Chang, Eur. Phys. J. C 75, 181 (2015)

54. L. Perivolaropoulos, arXiv:1104.0539

55. I. Antoniou, L. Perivolaropoulos, JCAP 1012, 012 (2012) 
56. G. Randers, Phys. Rev. 59, 195 (1941)

57. X. Li, Z. Chang, Phys. Rev. D 90, 064049 (2014)

58. A. Mazumdar, J. Rocher, Phys. Rep. 497, 85 (2011)

59. L. Wang, E. Pukartas, A. Mazumdar, JCAP 07, 019 (2013)

60. V. Mukhanov, Physical Foundations of Cosmology (Cambridge University Press, Cambridge, 2005)

61. H. Akbar-Zadeh, Acad. R. Belg. Bull. Cl. Sci. 74(5), 281 (1988)

62. A.R. Pullen, M. Kamionkowski, Phys. Rev. D 76, 103529 (2007). arXiv:0709.1144

63. X. Li, S. Wang (in progress)
64. A.E. Gumrukcuoglu, C.R. Contaldi, M. Peloso, JCAP 0711, 005 (2007)

65. M. Watanabe, S. Kanno, J. Soda, Mon. Not. R. Astron. Soc. 412, L83 (2011)

66. Z. Chang, S. Wang, arXiv:1312.6575 [astro-ph.CO]

67. R. Kothari, S. Ghosh, P.K. Rath, G. Kashyap, P. Jain, arXiv: 1503.08997

68. A. Lewis, A. Challinor, A. Lasenby, Astrophys. J. 538, 473 (2000)

69. C. Howlett, A. Lewis, A. Hall, A. Challinor, JCAP 04, 027 (2012) 